\title{
Diversité Des Espèces De Bois D'œuvre Menacées De Disparition De La Flore De La Côte d'Ivoire, Dans Le Massif Forestier Yapo-Abbé
}

\author{
Sié Fernand Pacôme Ouattara, Doctorant \\ Kouassi Kouadio, Maître de Conférences \\ Dodiomon Soro, Professeur Titulaire \\ Laboratoire de Botanique, UFR Biosciences,
} Université Félix Houphouët-Boigny, Côte d'Ivoire, Abidjan, Côte d'Ivoire

Doi:10.19044/esj.2019.v15n36p213 URL:http://dx.doi.org/10.19044/esj.2019.v15n36p213

Résumé

La présente étude a été réalisée dans le Massif Forestier Yapo-abbé (MFYA), au Sud-Est de la Côte d'Ivoire. Elle vise à évaluer la diversité des espèces de bois d'œuvre inscrites sur la liste rouge de l'UICN (2018). La méthode de relevé de surface a été utilisée pour l'inventaire et l'identification de ces espèces dans les trois blocs forestiers du massif. Les résultats montrent que le MFYA est riche de 22 espèces de bois d'œuvre inscrites sur la liste rouge de l'UICN (2018). Ces 22 espèces sont réparties en quinze espèces vulnérables $(68,2 \%)$, cinq espèces de préoccupation mineure $(22,8 \%)$, une espèce quasi-menacée $(4,5 \%)$ et une autre en danger de disparition $(4,5 \%)$. La fréquence d'apparition des espèces a permis d'obtenir trois groupes d'espèces : le groupe 1 (espèces constantes) compte une espèce, le groupe 2 (espèces accessoires) est constitué de six espèces ; quant au groupe 3 (espèces rares), il est composé de quinze espèces. Les trois biotopes du MFYA sont faiblement diversifiés, d'où la nécessité de les enrichir avec les espèces du groupe 3 , en particulier les blocs plantations forestières et jachères, pour une gestion durable dudit massif.

Mots clés: Diversité Floristique, Espèces De Bois D’œuvre, Liste Rouge De L'UICN, Massif Forestier Yapo-Abbé, Côte d'Ivoire 


\title{
Diversity of Timber Species Threatened of Disappearance of Côte d'Ivoire Flora, in the Yapo-Abbé Forest
}

\author{
Sié Fernand Pacôme Ouattara, Doctorant \\ Kouassi Kouadio, Maître de Conférences \\ Dodiomon Soro, Professeur Titulaire \\ Laboratoire de Botanique, UFR Biosciences,
}

Université Félix Houphouët-Boigny, Côte d'Ivoire, Abidjan, Côte d'Ivoire

\begin{abstract}
This study was conducted in Yapo-Abbe forest in South-Eastern Côte d'Ivoire. It aims to assess the diversity of timber species, listed on the IUCN Red List (2018). The surface survey method was used for the inventory and identification of these species in the three blocks of the forest. The results show that Yapo-Abbe forest is rich in 22 species of timber listed on the IUCN Red List (2018). These 22 species are divided into fifteen vulnerable species (68.2\%), five species of low concern (22.8\%), a near-threatened species $(4.5 \%)$ and another species at risk of extinction $(4.5 \%)$.). The frequency of occurrence of species has resulted in three groups of species; group 1 (constant species) has one species, group 2 (accessory species) consists of six species. As for group 3 (rare species), it's composed of fifteen species. The three biotopes of Yapo-Abbe forest are poorly diversified, hence the need to enrich them with the group 3 species, especially forest plantation and fallow blocks for sustainable management of this massif.
\end{abstract}

Keywords: Floristic Diversity, Timber Species, IUCN Red List, Yapo-Abbe Forest, Côte d'Ivoire

\section{Introduction}

La flore de la Côte d'Ivoire renferme selon Scouppe (2011), environ 4000 espèces de plantes supérieures de forêt et de savane. Considéré autrefois, comme l'un des écosystèmes tropicaux les plus diversifiés, le couvert forestier ivoirien est devenu aujourd'hui, l'un des plus menacés (Tuo et al., 2017). En effet, les activités humaines (l'exploitation forestière pour grumes, l'agriculture, l'élévage et l'urbanisation) ont entraîné la destruction de plus de $83 \%$ des surfaces forestières, occasionnant ainsi, la disparition de plusieurs 
espèces de plantes dont les espèces de bois d'œuvre (Aké-Assi, 1997 ; Konan et al., 2015). Comme conséquence, sur les 43 espèces de bois d'œuvre couramment exploitées pour la qualité technologique de leur bois, en Côte d'Ivoire, 26 sont inscrites sur la liste rouge de l'Union Internationale pour la Conservation de la Nature, version 2018, en abrégé UICN (2018). De ce fait, la production en volume de bois qui était de plus de 5 millions de $\mathrm{m}^{3}$ entre 1960 et 1977, a chuté à 2,5 millions de $\mathrm{m}^{3}$ entre 1980 et 1995 (Sattler, 1997). Aujourd'hui, cette production est moins de 2 millions de $\mathrm{m}^{3}$, si bien que les peuplements à diamètre exploitable de la quasi-totalité de ces espèces sont devenus rares (Kouadio, 2012). Or, la régénération naturelle de ces espèces de bois d'œuvre, inscrites sur la liste rouge de l'UICN, a été jugée faible par certains auteurs tels que Ettien (2005), dans la forêt classée du Haut-Sassandra et Kouadio et al. (2007), dans la forêt classée de Bossématié.

De nos jours, les Parcs Nationaux, les Réserves Biologiques et les Forêts Classées, constituent pour ces espèces d'importants refuges, malgré que ces Aires Protégées soient soumises à des pressions anthropiques sans précédent (pression démographique, activités agricoles clandestines et prélèvement des produits forestiers non ligneux). Cependant, le Massif Forestier Yapo-Abbé (MFYA), qui est l'une des 231 forêts classées en Côte d'Ivoire, semble être bien conservée du fait de sa richesse en espèces endémiques (126 espèces) et en espèces rares et/ou menacées d'extinction (51 espèces) de la flore ivoirienne (Konan, 2016). De ce fait, la probabilité de rencontrer les espèces de bois d'œuvre inscrites sur la liste rouge de l'UICN (2018), pourrait être élevée ; d'où l'intérêt de cette étude qui a pour but de constituer une base de données pour une gestion rationnelle et durable de cette forêt classée.

L’objectif général de cette étude est de mettre en évidence la richesse et la diversité des espèces de bois d'œuvre, inscrites sur la liste rouge de l'UICN (2018), dans le MFYA. De manière spécifique, il a été question de déterminer la richesse des trois blocs du MFYA, en espèces de bois d'œuvre inscrites sur la liste rouge de l'UICN (2018) et d'évaluer la diversité de ces espèces à travers les indices de diversité de Shannon, Simpson, Equitabilité et la fréquence spécifique, dans ces trois blocs.

\section{Materiel Et Methodes \\ Milieu d'étude}

L'étude a été réalisée dans le Massif Forestier Yapo-Abbé (MFYA), situé au Sud-Est de la Côte d'Ivoire, dans la région administrative de l'Agnéby-Tiassa, entre 5'40'02' ' et 5'47'32' ' de latitude Nord et 3'57'02' 'et 4'11'37' de longitude Ouest (Figure 1). D’une superficie de 28790 ha, le massif est à cheval sur les Sous-préfectures d'Azaguié (Département d'Agboville) et de Yakassé-Mé (Département d'Adzopé). Il est constitué de 
trois principaux blocs forestiers (Figure 1) : le bloc forêt secondaire (19001,4 ha), le bloc plantations forestières $(4318,5 \mathrm{ha})$ et le bloc jachère $(5470,1 \mathrm{ha})$. Le climat est de type subéquatorial, caractérisé par une pluviométrie moyenne d'environ $1400 \mathrm{~mm} /$ an et par une température moyenne annuelle de $27^{\circ} \mathrm{C}$ (SODEXAM, 2014). L'aspect général de la végétation du MFYA est du type forêt dense humide sempervirente, caractéristique du secteur ombrophile, d'après les subdivisions établies par Guillaumet et Adjanohoun (1971). Le relief est une pénéplaine, c'est-à-dire une succession ininterrompue de collines aux pentes faibles (moins de 5\%), de bas-fonds et de replats (SODEFOR, 1999). Sur le plan humain, le MFYA est à cheval sur les territoires des groupes ethniques Abbey et Attié des départements d'Agboville et d'Adzopé. A ces populations autochtones, s'ajoute une forte communauté d'allochtones et d'allogènes, qui a pour activité principale, l'agriculture (SODEFOR, 1999).

Le matériel d'étude est constitué des individus des espèces de bois d'œuvre, dont le diamètre est supérieure ou égale à $10 \mathrm{~cm}$.

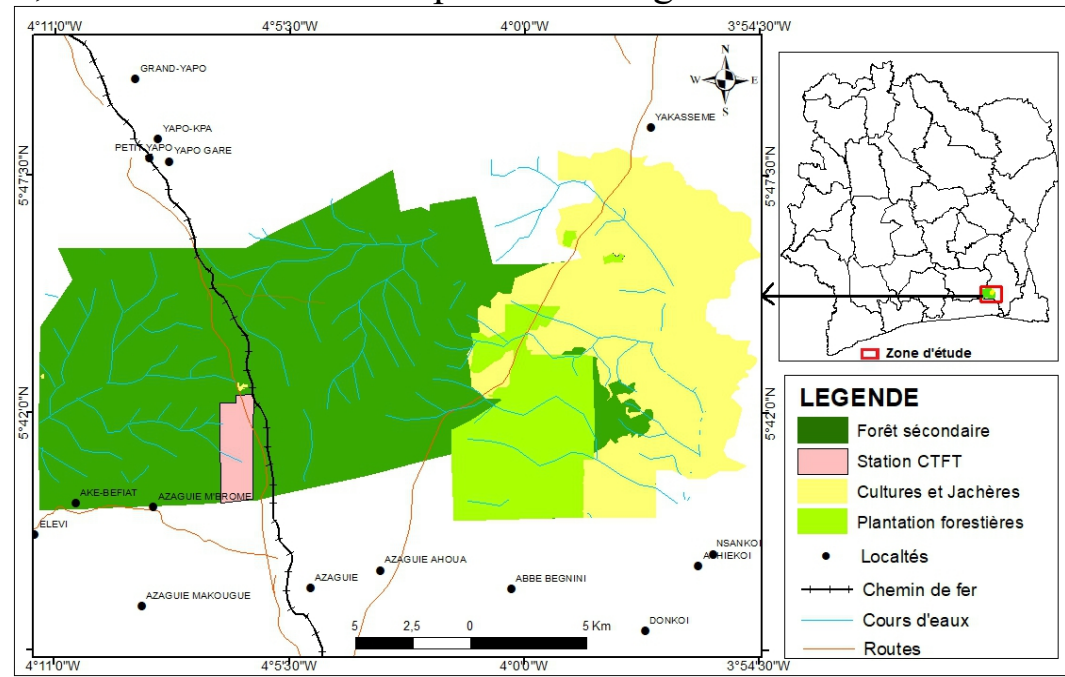

Figure 1 : Localisation du massif forestier Yapo-Abbé (source : Konan, 2016)

\section{Méthodes d'étude}

\section{Collecte de données}

L'inventaire floristique dans le Massif Forestier Yapo-Abbé (MFYA), a été réalisé à l'aide d'un placeau rectangulaire de $100 \mathrm{~m}$ de longueur et $50 \mathrm{~m}$ de largeur, soit une superficie de 0,5 ha. Au total, 60 placeaux ont été installés dans le MFYA, soit 20 placeaux par bloc (Figure 2). Dans chaque dispositif d'étude, toutes les espèces de bois d'œuvre de plus de $31,4 \mathrm{~cm}$ de circonférence ont été inventoriées et celles inscrites sur la liste rouge de l’UICN (2018), ont été géoréférencées. 


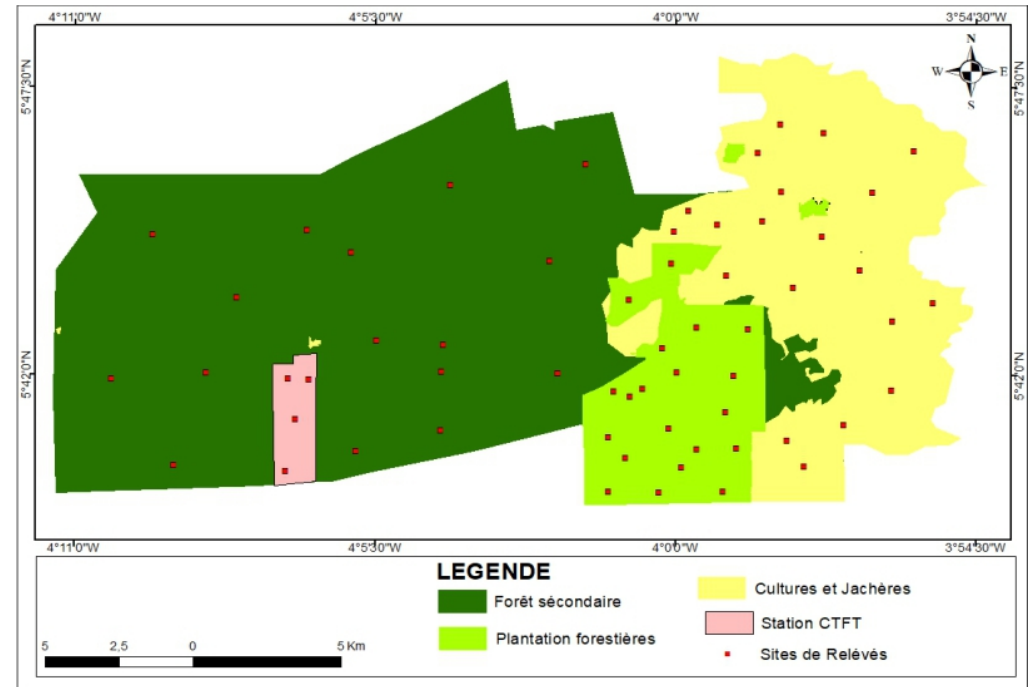

Figure 2: Carte de distribution des sites de relevé floristique dans le Massif ForestierYapo-Abbé

\section{Analyse des données \\ Richesse floristique}

Au cours de cette étude, la flore des espèces de bois d'œuvre inscrites sur la liste rouge de l'UICN (2018), a été établie. L'analyse qualitative (Familles, Genres, Espèces) a été réalisée à partir de la liste floristique établie pour chacun des différents blocs forestiers du massif. La nomenclature adoptée pour l'identification de ces espèces de bois d'œuvre est celle de APG III (2009) et APG IV (2016).

\section{Composition floristique}

L'analyse de la composition floristique a consisté à déterminer pour chacune des espèces de bois d'œuvre inscrites sur la liste rouge de l'UICN (2018), inventoriées au cours de cette étude, la catégorie selon la qualité technologique du bois (SODEFOR, 1992 ; Dupuy et al., 1997) et la valeur commerciale des espèces (P1: Espèces de première catégorie ou couramment commercialisées ; P2: Espèces de deuxième catégorie ou sporadiquement commercialisées et P3: Espèces de troisième catégorie ou à promouvoir), selon SODEFOR (1993). Pour la détermination des statuts de conservation de ces espèces, la liste rouge de l'UICN (2018), a servi de référence.

\section{Diversité floristique}

La diversité floristique a été évaluée à travers le coefficient de similitude de Sørensen (1948), les indices de Shannon et Weaver (1949), de Simpson (1949), d'Equitabilité de Piélou (1966) et la Fréquence d'apparition 
des espèces ou Fréquence spécifique. Pour cette étude, seules les espèces de bois d'œuvre inscrites sur la liste rouge de l'UICN (2018), ont été analysées.

\section{- Coefficient de similitude de Sørensen}

Le coefficient de similitude (Cs) a pour but de caractériser objectivement et quantitativement le degré de ressemblance entre deux listes floristiques de deux parcelles $\mathrm{A}$ et $\mathrm{B}$. Il permet de comparer deux à deux la liste floristique des parcelles afin de déterminer une ressemblance ou une dissemblance. Il existe plusieurs formules, mais celle de Sørensen (1948) a été adoptée dans le cadre de cette étude, pour comparer la ressemblance floristique des espèces de bois d'œuvre inscrites sur la liste rouge de l'UICN (2018), entre les différents blocs du MFYA. Le coefficient de similitude de Sørensen se calcule avec la formule mathématique suivante :

$$
\mathrm{Cs}=[\mathbf{2 C} /(\mathbf{a}+\mathbf{b})] \times 100
$$

$\mathrm{a}$ et $\mathrm{b}$ sont les nombres d'espèces appartenant respectivement aux parcelles $\mathrm{A}$ et $\mathrm{B}$;

c étant le nombre d'espèces communes aux deux parcelles. Le coefficient de similitude varie entre 0 et 100 . Lorsqu'il est supérieur ou égal à $50 \%$ (CS $\geq$ 50), alors l'hypothèse de ressemblance est admise. Dans le cas contraire, lorsque le coefficient de similitude est inférieur à 50\% (CS<50), il n’y a pas de ressemblance entre les listes floristiques des parcelles concernées.

\section{- Indice de diversité de Shannon et Weaver}

L'indice de diversité de Shannon permet de mesurer la composition en espèces d'un peuplement en combinant l'abondance relative des espèces et la richesse spécifique (Felfili et al., 2004). Cet indice est l'un des meilleurs estimateurs de la diversité biologique et il est utilisé comme mesure d'hétérogénéité. Dans la présente étude, il a été utilisé pour évaluer la diversité des trois blocs forestiers du MFYA, en espèces de bois d'œuvre inscrites sur la liste rouge de l'UICN (2018). Il se résume à l'expression mathématique suivante :

$$
\mathbf{H}^{\prime}=-\sum_{\mathbf{i}=\mathbf{1}}^{s} \mathbf{P}_{\mathbf{i}} \ln \left(\mathbf{P}_{\mathbf{i}}\right)
$$

avec $\mathrm{Pi}=\mathrm{Ni} / \mathrm{N}, \mathrm{P}_{\mathrm{i}}$ est la proportion d'une espèce $\mathrm{i}$ par rapport au nombre total d'espèces;

H' désigne l'indice de diversité de Shannon et s'exprime en bit.

Cet indice varie généralement entre 1 et 5 (Felfili et al., 2004). La diversité est faible lorsque H' est inférieur à 3 bits, moyenne si H' est compris entre 3 bits et 4 bits puis élevé quand H' est compris entre 4 et 5 . H' dépassant rarement les 5 bits. 


\section{- Indice de diversité de Simpson}

Quoique l'indice de Shannon soit couramment utilisé, il est nécessaire d'associer les différents indices de mesure de la diversité afin de s'assurer d'une même hiérarchisation entre les relevés floristiques (Scouppe, 2011). L'indice de diversité de Simpson (DS) permet de rendre compte de l'abondance d'une ou de quelques espèces. Plus la valeur de DS est élevée, plus le milieu à caractériser est hétérogène et la diversité forte. Sa formule mathématique est :

$$
\text { DS = } 1-\Sigma(\mathbf{N i}(\mathbf{N i}-1)) /(\mathbf{N}(\mathbf{N}-\mathbf{1}))
$$

DS représente l'indice de diversité de Simpson;

Ni est le nombre d'individus d'une espèce i;

$\mathrm{N}$ le nombre total d'individus de toutes les espèces. Dans cette étude, l'indice de diversité de Simpson a été utilisé pour caractériser l'hétérogénéité des différents blocs du MFYA, à travers la dominance d'une espèce (quand DS tend vers 0 ) ou la codominance de plusieurs espèces (quand DS tend vers 1).

\section{- Indice d'équitabilité de Piélou}

Cet indice, encore appelé indice de régularité ou d'équirépartition, renseigne sur la répartition des individus entre les différentes espèces (Adjakpa et al., 2013). L'indice d'équitabilité correspond au rapport entre la diversité de Shannon obtenue et la valeur de diversité maximale de Shannon qui est égale à 5 (Wala et al., 2005). Cet indice se calcule selon la formule mathématique suivante :

E représente l'équitabilité;

$$
\mathbf{E}=\mathbf{H} / \ln S
$$

H' l'indice de Shannon;

$\mathrm{S}$ le nombre total d'espèces sur une parcelle.

La valeur de cet indice tend vers 0 lorsqu'une espèce domine largement et est égale à 1 lorsque toutes les espèces ont la même abondance (Frontier et al., 2008). Dans cette étude, l'indice d'équitabilité de Piélou a été calculé pour apprécier l'équilibre de la répartition des individus des espèces de bois d'œuvre, inscrites sur la liste rouge de l'UICN (2018), dans les trois blocs forestiers du MFYA.

\section{- Fréquence d'apparition des espèces}

La fréquence d'apparition (Fr) d'une espèce ou fréquence spécifique, est le nombre d'occurrences de l'espèce ou encore le nombre de relevés où la présence de cette espèce est notée, sur le nombre total de relevés effectués (Dajoz, 2003 ; Silué, 2018). Elle exprime la constance d'une espèce dans les différentes parcelles d'un même habitat. Dans cette étude, la fréquence d'apparition a été calculée pour chacune des 22 espèces de bois d'œuvre 
inscrites sur la liste rouge de l'UICN (2018), à travers la formule mathématique suivante :

$$
\mathbf{F r}=\left(\mathbf{P}_{\mathrm{a}} / \mathbf{P}\right) \times 100
$$

$\mathrm{P}_{\mathrm{a}}$ représente le nombre de relevés contenant l'espèce prise en considération; $\mathrm{P}$ le nombre total de relevés.

Selon Dajoz (2003), on distingue trois groupes d'espèces : les espèces fréquentes ou constantes qui apparaissent dans au moins $50 \%$ des relevés ; les espèces peu fréquentes ou espèces accessoires apparaissent dans 25 à $49 \%$ des relevés et les espèces rares ou espèces accidentelles possédant une fréquence d'apparition inférieure à $25 \%$.

\section{Test de comparaison des moyennes}

Les valeurs moyennes calculées du nombre d'espèces inventoriées, des indices de Shannon, Simpson et Equitabilité, ont été analysées à l'aide du test non paramétrique de Kruskal Wallis. Ce choix est dû au fait que les conditions de normalité et d'homogénéité n'ont pas été remplies. Le test de Steel-DwassCritchlow-Fligner a été utilisé pour le classement des moyennes avec un seuil de probabilité $(\alpha=0,05)$. Le logiciel XLSTAT 2016.02 a permis de réaliser ce test.

\section{Resultats \\ Richesse floristique}

L'inventaire des trois blocs du Massif Forestier Yapo-Abbé (MFYA) a permis de recenser 49 espèces de bois d'œuvre dont 22 sont inscrites sur la liste rouge de l'UICN de 2018 (Tableau 1). Ces 22 espèces sont réparties entre 19 genres appartenant à 10 familles. Les Malvaceae et les Meliaceae sont les familles les plus importantes avec chacune 06 espèces, soit 27,27\% du total de ces espèces de bois d'œuvre inscrites sur la liste rouge de l'UICN de 2018. Elles sont suivies des Fabaceae et des Moraceae avec chacune 02 espèces, soit $9,09 \%$. 
Tableau 1 : Liste des espèces de bois d'œuvre inscrites sur la liste rouge de l'UICN (2018), recensées dans le Massif Forestier Yapo-Abbé

\begin{tabular}{|c|c|c|c|c|c|}
\hline $\mathrm{N}^{\circ}$ & Espèces & Familles & Noms communs & Catégories & $\begin{array}{l}\text { Statut uicn } \\
\text { (2018) }\end{array}$ \\
\hline 1 & Anopyxis klaineana (Pierre) Engl. & Rhizophoraceae & Bodioa & $\mathrm{P} 2$ & VU \\
\hline 2 & Berlinia confusa Hoyle & Fabaceae & Mélégba & $\mathrm{P} 2$ & $\mathrm{LC}$ \\
\hline 3 & Bombax brevicuspe Sprague & Malvaceae & Kondroti & $\mathrm{P} 1$ & VU \\
\hline 4 & Ceiba pentandra (L.) Gaertn. & Malvaceae & Fromager & $\mathrm{P} 1$ & $\mathrm{LC}$ \\
\hline 5 & $\begin{array}{l}\text { Entandrophragma } \quad \text { cylindricum } \\
\text { (Sprague) Sprague }\end{array}$ & Meliaceae & Adoudikro & $\mathrm{P} 1$ & VU \\
\hline 6 & $\begin{array}{l}\text { Entandrophragma angolense (Welw.) } \\
\text { C DC. }\end{array}$ & Meliaceae & Tiama & $\mathrm{P} 1$ & VU \\
\hline 7 & $\begin{array}{l}\text { Entandrophragma utile (Dawe \& } \\
\text { Sprague) Sprague }\end{array}$ & Meliaceae & Sipo & $\mathrm{P} 1$ & VU \\
\hline 8 & Eribroma oblongum (Mast.) Germain & Malvaceae & $\mathrm{Bi}$ & $\mathrm{P} 2$ & VU \\
\hline 9 & Guarea cedrata (A. Chev.) Pellegr. & Meliaceae & Bossé & P1 & VU \\
\hline 10 & Heritiera utilis Sprague & Malvaceae & Niangon & P1 & VU \\
\hline 11 & Khaya ivorensis A. Chev & Meliaceae & Acajou Bassam & $\mathrm{P} 1$ & VU \\
\hline 12 & Lophira alata Gaertn. f. & Ochnaceae & Azobé & $\mathrm{P} 1$ & VU \\
\hline 13 & Milicia excelsa (Welw.) C.C. Berg & Moraceae & Iroko brun & $\mathrm{P} 1$ & $\mathrm{LR} / \mathrm{nt}$ \\
\hline 14 & Milicia regia (A. Chev.) Berg & Moraceae & Iroko jaune & $\mathrm{P} 1$ & VU \\
\hline 15 & $\begin{array}{l}\text { Nauclea diderrichii (De Wild \& Th. } \\
\text { Dur.) Merill }\end{array}$ & Rubiaceae & Badi & $\mathrm{P} 1$ & VU \\
\hline 16 & $\begin{array}{l}\text { Nesogordonia papaverifera (A. Chev.) } \\
\text { Cap. }\end{array}$ & Malvaceae & Kotibé & $\mathrm{P} 1$ & VU \\
\hline 17 & Parinari excelsa Sabine & Chrysobalanaceae & Sougué & P3 & $\mathrm{LC}$ \\
\hline 18 & Parkia bicolor A. Chev. & Fabaceae & Lô & P3 & $\mathrm{LC}$ \\
\hline 19 & Terminalia ivorensis A. Chev. & Combretaceae & Framiré & $\mathrm{P} 1$ & VU \\
\hline 20 & Tieghemella heckelii A. Cher & Sapotaceae & Makoré & $\mathrm{P} 1$ & $\mathrm{EN}$ \\
\hline 21 & Triplochiton scleroxylon Schumann & Malvaceae & Samba & $\mathrm{P} 1$ & $\mathrm{LR} / \mathrm{lc}$ \\
\hline 22 & $\begin{array}{l}\text { Turraeanthus africanus (Welw.) } \\
\text { Pellegr. }\end{array}$ & Meliaceae & Avodiré & $\mathrm{P} 1$ & VU \\
\hline
\end{tabular}

$\mathrm{P} 1$ : espèces principales de $1^{\text {ère }}$ catégorie ; $\mathrm{P} 2$ : espèces principales de $2^{\text {ème }}$ catégorie $; \mathrm{P} 3:$ espèce principale de $3^{\text {ème }}$ catégorie ; EN: En danger de disparition ; VU: Vulnérable ; LR/nt : Quasi-menacées ; LC et LR/Ic : préoccupation mineure.

Le nombre d'espèces inventoriées de bois d'œuvre inscrites sur la liste rouge de l'UICN de 2018, varie de 13 à 21 dans les trois blocs forestiers du massif (Tableau 2). Le nombre moyen de ces espèces recensées dans les placeaux, est de 4,45 pour le bloc forêt secondaire ; 3,20 pour le bloc plantations forestières et 2,45 pour le bloc jachère. Les résultats du Tableau 2 ont montré qu'il n'y a pas de différence significative entre ces valeurs moyennes $(K=5,561 ; P=0,062)$. 
Tableau 2 : Richesse en espèces de bois d'œuvre inscrites sur la liste rouge de l'UICN (2018), avec leurs valeurs moyennes dans les différents blocs du Massif Forestier Yapo-Abbé

\begin{tabular}{ccccc}
\hline Biotopes & $\begin{array}{c}\text { Nombre } \\
\text { de Familles }\end{array}$ & $\begin{array}{c}\text { Nombre } \\
\text { de Genres }\end{array}$ & $\begin{array}{c}\text { Nombre } \\
\text { d'Espèces }\end{array}$ & $\begin{array}{c}\text { Nombre moyen } \\
\text { d'espèces }\end{array}$ \\
\hline $\begin{array}{c}\text { Forêt secondaire } \\
\text { Plantations }\end{array}$ & 11 & 18 & 21 & $4,45 \pm 2,8^{\mathrm{a}}$ \\
forestières & 11 & 14 & 16 & $3,20 \pm 1,47^{\mathrm{a}}$ \\
Jachères & 9 & 13 & 13 & $2,45 \pm 1,23^{\mathrm{a}}$ \\
MFYA & 12 & 19 & 22 & - \\
\hline
\end{tabular}

Les valeurs moyennes accompagnées de même lettre ne sont pas significativement différentes au seuil de $\alpha=5 \%$

\section{Composition floristique}

En considérant les catégories commerciales des espèces de bois d'œuvre, les 22 espèces inventoriées dans le MFYA sont représentées par 17 P1, soit 77,27\% ; 03 P2 (13,64\%) et 02 P3 (9,09\%). Les trois blocs du MFYA sont plus peuplés des espèces de première catégorie $\mathrm{P} 1$, avec une importance remarquable dans le bloc forêt secondaire (Figure 3).

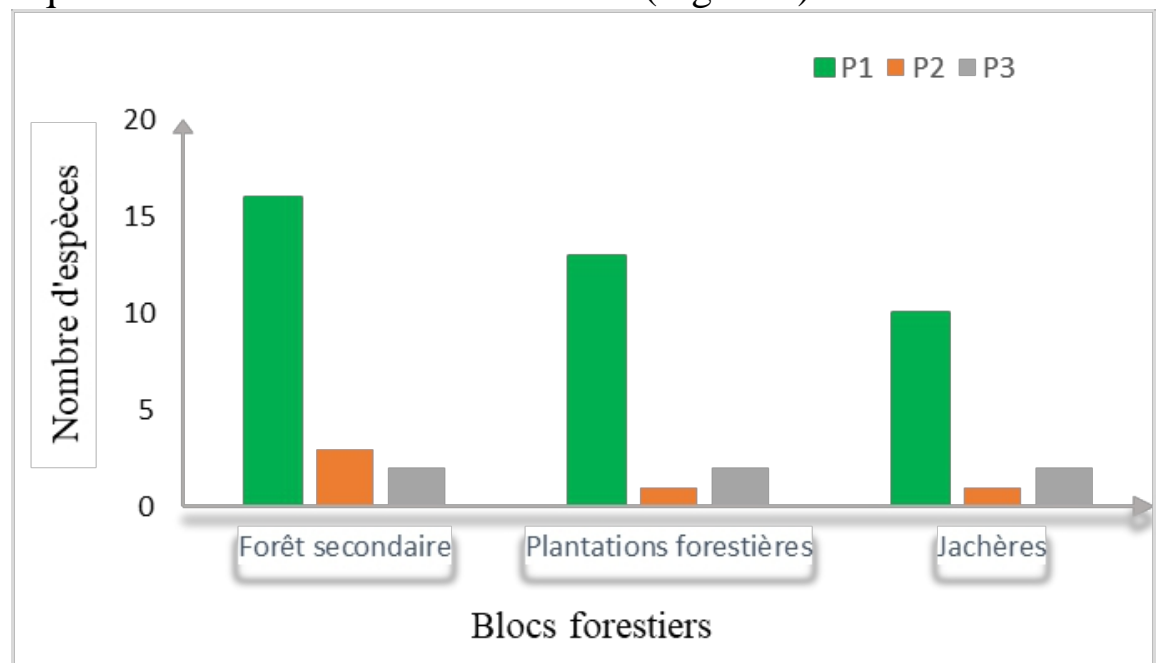

Figure 3 : Distribution des catégories commerciales des espèces de bois d'œuvre inscrites sur la liste rouge de l'UICN (2018), dans les blocs du Massif Forestier Yapo-Abbé

Concernant les différents statuts de conservation de l'UICN, ces 22 espèces de bois d'œuvre inscrites sur la liste rouge comptent quinze espèces vulnérables (VU), soit $68,2 \%$ contre cinq espèces qualifiées de préoccupation mineure (LC ou LR/Ic), soit $(22,8 \%)$. Les espèces quasi-menacées (LR/nt) avec $4,5 \%$ et celles en danger de disparition (EN) avec également $4,5 \%$, sont représentées chacune par une seule espèce. 


\section{Diversité floristique}

\section{Ressemblance floristique entre les blocs du Massif Forestier Yapo-Abbé}

Les valeurs de l'indice de similarité de Sørensen calculées (Tableau 3), sont de $81,08 \%$ pour le couple (forêt secondaire - plantations forestières), $70,58 \%$ pour le couple (forêt secondaire-jachères) et $82,75 \%$ pour le couple (plantations forestières-jachères). Ces valeurs indiquent que les trois blocs du MFYA sont floristiquement identiques.

Tableau 3 : coefficient de similitude de Sorensen entre les biotopes du massif forestier Yapo-Abbé

\begin{tabular}{llll}
\hline Biotopes & Forêt secondaire & $\begin{array}{l}\text { Plantations } \\
\text { forestières }\end{array}$ & Jachères \\
\hline Forêt secondaire & - & 81,08 & 70,58 \\
Plantations & & - & 82,75 \\
forestières & & & - \\
Jachères & & & - \\
\hline
\end{tabular}

\section{Diversité spécifique des blocs du Massif Forestier Yapo-Abbé}

Les valeurs totales de l'indice de Shannon calculées sont de 1,6 bits pour le bloc plantations forestières ; 2,1 bits pour le bloc jachère et 2,3 bits pour le bloc forêt secondaire (Tableau 4). Ces valeurs montrent que les trois biotopes du Massif Forestier Yapo-Abbé (MFYA) sont faiblement diversifiés en espèces de bois d'œuvre inscrites sur la liste rouge de l'UICN version 2018. Les résultats de l'analyse statistique dans le Tableau 4, indiquent que les valeurs moyennes de l'indice de Shannon des trois blocs forestiers du massif, sont statistiquement égales $(K=1,281 ; P=0,527)$.

Concernant l'indice de Simpson, les valeurs totales obtenues pour les biotopes forêt secondaire $(0,8)$ et jachère $(0,9)$ tendent vers 1 (Tableau 4$)$. Ces valeurs totales indiquent que ces deux biotopes sont assez hétérogènes, tandis que le biotope plantations forestières, avec une valeur d'indice de Simpson de 0,6 est moins diversifié en espèces de bois d'œuvre inscrites sur la liste rouge de l'UICN de 2018. Les résultats de l'analyse statistique (Tableau 4) démontrent cette hétérogénéité des blocs forêt secondaire et jachère, avec des valeurs moyennes d'indice de Simpson significativement différentes $(K=6,323 ; P=0,042)$ de celle du bloc plantations forestières.

Quant à l'indice d'équitabilité de Piélou, les valeurs totales obtenues sont de 0,6 pour le bloc plantations forestières ; 0,7 pour le bloc forêt secondaire et 0,8 pour les jachères (Tableau 4). Ces valeurs montrent que, la répartition des individus des 22 espèces de bois d'œuvre menacées de disparition selon l'UICN, est relativement plus équilibrée dans le biotope jachère que dans les deux autres biotopes (forêt secondaire et plantations forestières). Cependant, les résultats de l'analyse statistique consignés dans le Tableau 4, montrent que les valeurs moyennes de cet indice, déterminées pour 
chacun des trois blocs forestiers du massif, ne sont pas significativement différentes $(K=4,937 ; P=0,085)$.

Tableau 4 : Valeurs des indices de diversité des trois blocs du massif forestier Yapo-Abbé

\begin{tabular}{lllllll}
\hline Biotopes & \multicolumn{2}{l}{ Indice Shannon } & \multicolumn{2}{l}{ Indice Simpson } & \multicolumn{2}{l}{ Equitabilité } \\
& $\begin{array}{l}\text { Valeurs } \\
\text { totales }\end{array}$ & $\begin{array}{l}\text { Valeurs } \\
\text { moyennes }\end{array}$ & $\begin{array}{l}\text { Valeurs } \\
\text { Totales }\end{array}$ & $\begin{array}{l}\text { Valeurs } \\
\text { moyennes }\end{array}$ & $\begin{array}{l}\text { Valeurs } \\
\text { totales }\end{array}$ & $\begin{array}{l}\text { Valeurs } \\
\text { moyennes }\end{array}$ \\
\hline $\begin{array}{l}\text { Forêt } \\
\text { secondaire }\end{array}$ & 2,3 & $0,91 \pm 0,66^{\mathrm{a}}$ & 0,8 & $0,67 \pm 0,31^{\mathrm{ab}}$ & 0,7 & $0,60 \pm 0,38^{\mathrm{a}}$ \\
$\begin{array}{l}\text { Plantations } \\
\text { forestières }\end{array}$ & 1,6 & $0,77 \pm 0,45^{\mathrm{a}}$ & 0,6 & $0,56 \pm 0,29^{\mathrm{a}}$ & 0,6 & $0,65 \pm 0,30^{\mathrm{a}}$ \\
Jachères & 2,1 & $0,75 \pm 0,43^{\mathrm{a}}$ & 0,9 & $0,77 \pm 0,26^{\mathrm{b}}$ & 0,8 & $0,78 \pm 0,35^{\mathrm{a}}$ \\
\hline
\end{tabular}

Les valeurs moyennes affectées de lettres différentes pour une même colonne sont statistiquement différentes au seuil $5 \%$ et celles affectées de mêmes lettres, ne sont pas significativement différentes

\section{Fréquence d'apparition des espèces dans le massif forestier Yapo-Abbé}

L'évaluation de la fréquence d'apparition des 22 espèces de bois d'œuvre inscrites sur la liste rouge de l'UICN version 2018, dans le MFYA, montre trois groupes d'espèces (Tableau 5). Le groupe 1 correspondant aux espèces fréquentes ou constantes, comporte une seule espèce (Heritiera utilis), avec un taux d'apparition de 56,66\%. Le groupe 2 est composé de six espèces peu fréquentes ou accessoires dont la fréquence varie entre 25 et $35 \%$. Les espèces rares ou accidentelles constituant le groupe 3 , comptent quinze espèces avec une fréquence d'apparition qui varie entre 1,66 et $16,66 \%$. Ces valeurs indiquent que Heritiera utilis est l'espèce la plus rencontrée dans le MFYA, tandis que quinze autres espèces y sont très faiblement représentées. De plus, dans cette forêt classée, aucune espèce de bois d'œuvre inscrite sur la liste rouge de l'UICN de 2018, n'a été rencontrée à la fois dans les 60 placeaux installés.

Tableau 5: Fréquence d'apparition et statut des espèces de bois d'œuvre inventoriées et inscrites sur la liste rouge de l'UICN, dans le massif forestier Yapo-Abbé

$\begin{array}{lll}\text { Statut des espèces } & \text { Espèces recensées } & \text { Fréquence spécifique }(\%)\end{array}$

\begin{tabular}{lll}
\hline Espèces constantes & Heritiera utilis & 56,66 \\
\hline \multirow{4}{*}{ Espèces peu fréquentes } & Parkia bicolor & 35 \\
& Milicia excelsa & 28,33 \\
& Entandrophragma angolense & 26,66 \\
& Khaya ivorensis & 26,66 \\
& Terminalia ivorensis & 26,66 \\
& Bombax brevicuspe & 25 \\
\hline \multirow{3}{*}{ Espèces rares } & Parinari excelsa & 16,66 \\
& Entandrophragma utile & 15 \\
& Eribroma oblogum & 13,33
\end{tabular}




$\begin{array}{ll}\text { Berlinia confusa } & 10 \\ \text { Ceiba pentandra } & 10 \\ \text { Guarea cedrata } & 8,33 \\ \text { Tieghemella heckelii } & 8,33 \\ \text { Anopyxis klaineana } & 6,66 \\ \text { Nauclea diderrichii } & 6,66 \\ \text { Lophira alata } & 5 \\ \text { Milicia regia } & 5 \\ \text { Entandrophragma cylindricum } & 1,66 \\ \text { Nesogordonia papaverifera } & 1,66 \\ \text { Triplochiton scleroxylon } & 1,66 \\ \text { Turraeanthus africanus } & 1,66\end{array}$

\section{Discussion}

Les blocs forêt secondaire et plantations forestières du Massif Forestier Yapo-Abbé (MFYA), sont relativement plus peuplés en espèces de bois d'œuvre inscrites sur la liste rouge de l'UICN (2018) que le bloc jachère. La forte présence de ces espèces dans la forêt secondaire est imputable à la stabilité de ce biotope. En effet, c'est le bloc le mieux conservé du MFYA et constitue de ce fait, une zone de protection pour ces espèces, comme l'a souligné Konan (2016). La faible présence des espèces de bois d'œuvre inscrites sur la liste rouge de l'UICN (2018) dans le bloc jachère, pourrait s'expliquer par la présence humaine quasi-permanente et l'installation de plantations agricoles, qui ont occasionné la destruction de ces espèces dans ledit biotope.

Le nombre d'espèces de bois d'œuvre inscrites sur la liste rouge de l'UICN (2018), recensées au cours de cette étude, est élevé par rapport à celui obtenu par Konan (2016), dans le même massif. En effet, ce dernier, au cours de ces travaux, a révélé dix-sept espèces de d'œuvre inscrites sur la liste rouge de l'UICN. Cette différence peut s'expliquer soit par la taille de la surface échantillonnée, qui est de 30 ha pour cette étude contre 25 ha utilisés par Konan (2016); soit par le fait que la liste rouge de l'UICN a connu une augmentation du nombre d'espèces de bois d'œuvre, passant de 30 en 2015 à 36 en 2018.

Aussi, les résultats de cette étude sont-ils supérieurs à ceux obtenus par Koffi et al. (2015), au cours de leurs travaux dans la Forêt des Marais TanoéEhy (FMTE), située dans le Sud-Est du pays tout comme le MFYA. Cette différence floristique est probablement liée à la typologie de ces forêts. Alors que le MFYA est une forêt de terre ferme, la FMTE est une forêt marécageuse dont certaines espèces végétales s'y développent difficilement.

Par ailleurs, le nombre d'espèces de bois d'œuvre inscrites sur la liste rouge de l'UICN (2018) enregistré dans le MFYA, est inférieur à celui obtenu 
(28) par Kouadio (2007) dans la forêt classée de Bossématié, à l'Est du pays. Cette différence pourrait être liée à l'utilisation de différentes méthodes de collecte de données. En effet, au cours de son étude, Kouadio (2007) a combiné les méthodes itinérante et de relevé de surface, tandis que la dernière a seulement été utilisée dans le cadre de cette étude.

La faible présence des catégories $\mathrm{P} 2$ et $\mathrm{P} 3$ parmi les 22 espèces de bois d'œuvre inscrites sur la liste rouge de l'UICN (2018), recensées dans le MFYA, montre que les espèces de la catégorie $\mathrm{P} 1$ sont les plus menacées. En effet, en raison de la bonne qualité technologique de leur bois, ces espèces de première catégorie demeurent les plus exploitées (SODEFOR, 1993 ; Kouadio et al., 2018). Cela signifie aussi que les espèces utilisées pour l'enrichissement et le reboisement du massif, appartiennent essentiellement à la catégorie P1.

La présence des différents groupes d'espèces à statut UICN dans le MFYA, notamment celui des espèces en danger de disparition, témoigne de la stabilité et de la bonne conservation de ce massif, malgré les agressions qu'il subit. La ressemblance floristique relevée entre les trois biotopes du MFYA, indique qu'au cours des différents travaux antérieurs d'aménagement, aucun bloc n'a été privilégié par rapport aux deux autres. Ces travaux d'enrichissement et de reboisement dans les trois blocs, ont été réalisés pratiquement avec les mêmes espèces de bois d'œuvre. Cependant, les indices de diversité montrent que les trois biotopes du MFYA sont faiblement et inégalement diversifiés. Il nécessite donc, de les renforcer davantage en espèces de bois d'œuvre inscrites sur la liste rouge de l'UICN (2018), par les gestionnaires de cette forêt classée.

Or, la fréquence d'apparition de ces espèces indique la présence d'un nombre important d'espèces rares ou accidentelles; d'où l'intérêt d'enrichir les biotopes les moins fournis avec ces espèces rares, notamment les blocs plantations forestières et jachère. Les espèces qui pourraient être prioritairement utilisées sont : Tieghemella heckelii (P1, EN), Entandrophragma cylindricum (P1, VU), Entandrophragma utile (P1, VU), Guarea cedrata (P1, VU) Nauclea diderrichii (P1, VU), Lophira alata (P1, VU) et Turraeanthus africanus (P1, VU). Ces espèces se développent mieux en zone humide sempervirente alors que les espèces telles que Nesogordonia papaverifera, Milicia regia et Triplochiton scleroxylon, qui sont également des espèces rares dans le massif, se développent mieux en zone humide semidécidue.

\section{Conclusion}

L'inventaire réalisé dans le Massif Forestier Yapo-Abbé (MFYA) a permis de recenser 49 espèces de bois d'œuvre dont 22 sont inscrites sur la liste rouge de l'UICN (2018). Le MFYA est faiblement diversifié en espèces de bois d'œuvre inscrites sur la liste rouge de l'UICN (2018) et la répartition 
des individus de ces espèces n'est pas assez équilibrée dans l'ensemble du massif. La fréquence d'apparition des espèces a montré une forte proportion des espèces rares ou accidentelles dans le MFYA. Il serait souhaitable pour les gestionnaires de cette forêt classée, d'envisager un enrichissement des biotopes, surtout les moins diversifiés, avec ces espèces rares, notamment Tieghemella heckelii, Entandrophragma cylindricum, Entandrophragma utile, Nauclea diderrichii, Lophira alata et Turraeanthus africanus. Sur les 36 espèces de bois d'œuvre ivoiriennes inscrites sur la liste rouge de l'UICN (2018), 22 ont été rencontrées dans le MFYA. Les quatorze autres espèces non rencontrées pourraient être utilisées pour l'enrichissement de cette forêt, afin d'accroître sa richesse floristique et par conséquent sa biodiversité végétale.

\section{References:}

1. Adjakpa, B. J., Yedomonhan, H., Ahoton, L. E., Weesie, P. D. M., Akpo, E. L. 2013. Structure et diversité floristique des îlots de forêts riveraines communautaires de la vallée de Sô du Bénin. J. Appl. Biosci. 65: 4902- 4911.

2. Aké-Assi, L. 1997. Préface. In: SATTLER D. Eds. Bois de Côte d'Ivoire: Précis de reconnaissance des arbres commercialisés. Abidjan, Côte d'Ivoire: CEDA, pp. 7-9.

3. APG III. 2009. An update of the angioperm phylogeny groupe classification for the orders and families of floweringing plants. Bot. J. Linn. Soc. 161:105-121.

4. APG IV. 2016. An update of the Angiosperm Phylogen Group classification for the orders and families of flowering plants. Botanical Journal of the Linnean Society, 181: 1-20.

5. Beaufort, W. H. J. 1972. Distribution des arbres en forêt sempervirente de Côte d'Ivoire, ORSTOM, 48p.

6. Dajoz, R. 2003. Précis d'écologie. Dunod, Paris, France, 615 p.

7. Dupuy, B., Doumbia, F., Diahuissie, A., \& Brevet, R. 1997. Effet de types d'éclaircie en forêt dense humide ivoirienne. Bois Forêt Trop. 253 (3): 5-18.

8. Ettien, D. T. 2005. Potentiels de régénération des essences forestières commerciales par la germination des graines, dans la forêt classée du Haut-Sassandra (Centre-Ouest de la Côte d'Ivoire). Thèse de Doctorat 3 ème cycle, UFR Biosciences, Université de Cocody, Abidjan, Côte d'Ivoire, $259 \mathrm{p}$.

9. Felfili, J. M., Silva Júnior, M. C., Sevilha, A. C., Fagg, C. W., Walter, B. M. T., Nogueira, P. E., \& Rezende, A. V. 2004. Diversity, floristic and structural patterns of cerrado vegetation in Central Brazil. Plant Ecology 175: 37 - 46. 
10. Frontier, S., Pichod-Viale, D., Leprêtre, A., Davoult, D., Luczak, C. 2008. Écosystèmes: structure, fonctionnement, évolution. 4è édition. Dunod, Paris, France, 558 p.

11. Guillaumet, J. L., \& Adjanohoun, E. 1971. La végétation de la Côte d'Ivoire. In : Le Milieu Naturel de la Côte d'Ivoire. ORSTOM, Paris, France : 161-262.

12. Koffi, M., Ouattara, D. N., Koné, M., Bakayoko, A. 2015. Étude floristique et diversité de la forêt des Marais Tanoé-Ehy (Sud- Est de la Côte d'Ivoire). Journal of Animal \& Plant Sciences 25(3) : 39173938.

13. Konan, D., Bakayoko, A., Tra Bi, F. H., Bitignon, B. G. A., \& Piba, S. C. 2015. Dynamisme de la Structure diamétrique du peuplement ligneux des différents biotopes de la forêt classée de Yapo-Abbé, Sud de la Côte d'Ivoire. Journal of Applied Biosciences, 94: 8869-8879.

14. Konan, D. 2016. Etude de la dynamique floristique, structurale et du potentiel germinatif du stock semencier du sol de la forêt classée de yapo abbé : contribution pour une gestion durable des forêts classées de la Côte d'Ivoire. Thèse Unique, UFR des Sciences de la Nature, Université Nangui Abrogoua, Abidjan, Côte d'Ivoire, 217 p.

15. Kouadio, K. 2007. Etudes de la flore, de la végétation et impact de la l'éclaircie sélective, par dévitalisation, sur la croissance en épaisseur des essences principales de la forêt classée de Bossématié, dans l'est de la Côte d'Ivoire. Thèse de Doctorat d'Université de Cocody Abidjan, Côte d'Ivoire, $197 \mathrm{p}$.

16. Kouadio, K., Kouassi, K. E., Kouamé, N. F., \& Traoré, D. 2007. Evaluation des effets de l'éclaircie sélective, par dévitalisation, sur la croissance en diamètre des essences principales dans la forêt classée de Bossématié (Côte d'Ivoire). Agron. Afr. XIX (1) : 1-12.

17. Kouadio, K. 2012. Etude du comportement des essences locales exploitées couramment et menacées de disparition, en essai de reboisement dans la pépinière de l'UGF de Bossématié, en fonction de l'intensité de l'ensoleillement. Rapport Final du projet d'étude, Université Abidjan-Cocody, Côte d'Ivoire, $57 \mathrm{p}$.

18. Piélou, E. C. 1966. Species diversity and pattern diversity in the study of ecological succession. Journal of Theoritical Biology 10: 370-383.

19. Sattler, D. 1997. Bois de Côte d'Ivoire : précis de reconnaissance des arbres commercialisés. CEDA, Abidjan, Côte d'ivoire, $387 \mathrm{p}$.

20. Scouppe, M. 2011. Composition floristique et diversité de la végétation de la zone Est du Parc National de Taï (Côte d'Ivoire). Master Université de Genève, 194 p.

21. Shannon, C. E., Weaver, W. 1949. The mathematic theory of communications. University Illinois Press, Urbana, 117 p. 
22. Silué, P. A. 2018. Etudes de la flore, de la dynamique de la végétation et essai de germination de trois espèces couramment exploitées des forêts classées de la Palé et de Pouniakélé, dans la région de la Bagoué (Nord-Ouest de la Côte d'Ivoire). Thèse Unique, UFR Biosciences, Université de Félix Houphouët-Boigny, Abidjan, Côte d'Ivoire, 202 p. 23. Simpson, E. H. 1949. Measurement of diversity. Nature 163: 160-163.

24. SODEFOR. 1992. Inventaire en forêt dense pour la préparation d'un aménagement : inventaire et aménagement (première partie). Rapport scientifique, SODEFOR, Abidjan, Côte d'Ivoire, $16 \mathrm{p}$.

25. SODEFOR. 1993. Règles de culture et d'exploitation en forêt dense de Côte d'Ivoire. Rapport scientifique, SODEFOR, Abidjan, Côte d'Ivoire, $54 \mathrm{p}$.

26. SODEFOR. 1999. Plan d'aménagement de la forêt classée de YapoAbbé : 1999-2023, SODEFOR Edition, 141 p.

27. SODEXAM. 2014. Données météorologiques de la région de l'Agnéby-Tiassa.

28. Sørensen, T. 1948. A method of establishing group of equal amplitude in plant sociology based on similarity of species content and its application to analyse of the vegetation on danish common. Kjöbenhavn 4: 1-34.

29. Tuo N. F., Koffi, K. J., Kouassi, A. F., Koné, M., Adama, B., Bogaert, J. 2017. Etude de la diversité, de l'endémisme et de la distribution spatiale des Rubiaceae de Côte d'Ivoire. International Journal of Biological and Chemical Sciences 11(2): 777-797.

30. UICN. 2018. IUCN Red List of Threatened Species. www.iucnreadlist.org. Visité le 27/02/2018.

31. Wala, K., Sinsin, B., Guelly, K. A., Kokou, K., Akpagana, K. 2005. Typologie et structure des parcs agroforestiers de la préfecture de Doufelegou (Togo). Sécheresse 16: 209-216. 